S.-Y. Kim

Nagoya Math. J.

Vol. 189 (2008), 155-168

\title{
ANALYTIC JET PARAMETRIZATION FOR CR AUTOMORPHISMS OF SOME ESSENTIALLY FINITE CR MANIFOLDS
}

\author{
SUNG-YEON KIM
}

\begin{abstract}
In this paper we construct analytic jet parametrizations for the germs of real analytic CR automorphisms of some essentially finite CR manifolds on their finite jet at a point. As an application we show that the stability groups of such CR manifolds have Lie group structure under composition with the topology induced by uniform convergence on compacta.
\end{abstract}

Let $(M, p)$ be the germ of a real analytic CR manifold in $\mathbb{C}^{N}$. An important biholomorphic invariant of $(M, p)$ is the stability group $\operatorname{Aut}(M, p)$, i.e. the set of germs of biholomorphic maps $h:\left(\mathbb{C}^{N}, p\right) \rightarrow\left(\mathbb{C}^{N}, p\right)$ such that $h(M) \subset M$. Aut $(M, p)$ is a topological group under composition with the topology induced by uniform convergence on compacta, i.e. a sequence $\left\{h_{j}\right\} \subset \operatorname{Aut}(M, p)$ converges to $h \in \operatorname{Aut}(M, p)$ if there exists a neighborhood $U$ of $p$ such that all $h_{j}$ and $h$ are well-defined in $U$ and $h_{j}$ converges uniformly to $h$ on any compact set $K \subset U$.

In [9], Chern and Moser showed that the germ of a biholomorphism of $\mathbb{C}^{N}$ which preserves a nondegenerate real hypersurface $(M, p)$ is determined by its 2 -jet at $p$. Moreover, the dependence on the jet is real analytic. Therefore $\operatorname{Aut}(M, p)$ is a Lie group.

A generalization of the nondegeneracy condition on the Levi form is a certain nondegeneracy condition on the higher order Segre map at a reference point (see the definition in Section 1 ). We say that $(M, p)$ is finitely nondegenerate if the $k$-th Segre map at $p$ is an embedding near $p$ for some $k$. $(M, p)$ is Levi nondegenerate if and only if the first Segre map at $p$ is an embedding near $p([20])$. We say that $(M, p)$ is essentially finite if the $k$-th Segre map at $p$ is a finite to one map near $p$ for some $k$. Since the Segre map of any order is a holomorphic map, $(M, p)$ is essentially finite if the $k$-th Segre map at $p$ is a finite branched holomorphic covering onto its

Received November 1, 2005.

Revised March 10, 2007.

2000 Mathematics Subject Classification: 32V40, 32V20. 
image for some $k$.

The existence of an analytic jet parametrization for real analytic CR automorphisms of $(M, p)$ and the existence of a Lie group structure on the stability group are shown in case when $(M, p)$ is of finite type in the sense of Kohn-Bloom-Graham ([17], [8]) and finitely nondegenerate by the method of Segre varieties in [2], [3], [5], [15], [18], [21] or by the method of prolongations of CR embedding equations for hypersurface case in [12], [13], [14]. The finite determination for real analytic CR automorphisms is proved in case when $(M, p)$ is of finite type and essentially finite in [6].

Let $f$ be the germ of a holomorphic map. We denote by $j_{Z}^{k} f$ the $k$-jet of $f$ at $Z$ and by $J^{k}\left(\mathbb{C}^{N}, \mathbb{C}^{N^{\prime}}\right)$ the $k$-jet bundle of holomorphic maps from $\mathbb{C}^{N}$ to $\mathbb{C}^{N^{\prime}}$.

TheOREm 1. Let $(M, p)$ be the germ of a real analytic CR manifold in $\mathbb{C}^{N}$. Suppose $(M, p)$ is of finite type. Suppose further that there exists a positive integer $k_{0}$ such that the $k_{0}$-th Segre map of $M$ at $p$ is a finite branched holomorphic covering onto its image with critical set of codimension greater than or equal to 2 at $p$.

Then there exists an integer $k$ such that for any germ of a local biholomorphic map $f:\left(\mathbb{C}^{N}, p\right) \rightarrow\left(\mathbb{C}^{N}, p\right)$ with $f(M) \subset M$, there exist a neighborhood $U$ of $p$ in $\mathbb{C}^{N}$, a neighborhood $V$ of $\left(p, j_{p}^{k} f\right)$ in $J^{k}\left(\mathbb{C}^{N}, \mathbb{C}^{N}\right)$ and a holomorphic map $\Phi$ defined in $U \times V$ such that for any $q \in M \cap U$ and for any germ of a holomorphic map $h$ at $q$ such that $\left(q, j_{q}^{k} h\right) \in V$ and $h(M) \subset M$, we have

$$
h(Z)=\Phi\left(Z, q, j_{q}^{k} h\right)
$$

for all $Z$ sufficiently close to $q$.

Let $G_{p}^{k}\left(\mathbb{C}^{N}\right)$ be the set of $k$-jets of holomorphic maps $f:\left(\mathbb{C}^{N}, p\right) \rightarrow$ $\left(\mathbb{C}^{N}, p\right)$ which are invertible. Then $G_{p}^{k}\left(\mathbb{C}^{N}\right)$ is a complex Lie group under composition.

Corollary 2. Let $(M, p)$ and $k$ be as in Theorem 1 . Then the jet evaluation map

$$
j_{p}^{k}: \operatorname{Aut}(M, p) \longrightarrow G_{p}^{k}\left(\mathbb{C}^{N}\right)
$$

is a homeomorphism onto a totally real Lie subgroup of $G_{p}^{k}\left(\mathbb{C}^{N}\right)$. 
This corollary is a special case of [19], where it is shown that Corollary 2 is true for any germ of real analytic generic essentially finite minimal CR manifold $(M, p)$ and sufficiently large $k$. In that paper, authors showed the finite jet determination for CR automorphisms of $M$. For global Lie group structure on Aut $(M)$, Baouendi-Rothschild-Winkelmann-Zaitsev showed in [7] that a closed subgroup of analytic automorphisms having an analytic jet parametrization has a Lie group structure. From this and Theorem 1, we have a global result as follows.

Corollary 3. Let $M$ be a real analytic CR manifold. Suppose that there exists an integer $k$ such that at any $p \in M$, the germ $(M, p)$ satisfies the condition in Theorem 1. Then the group of CR automorphisms of $M$ has a Lie group structure with topology induced by uniform convergence on compacta.

Note that finite nondegeneracy condition is stronger than the condition in Theorem 1 and that the condition on the codimension of the critical set of the Segre map is essential to have an analytic jet parametrization: We will give examples of each case in Section 3.

The main technique of this paper is to construct so called 'Basic identity of reflection type' for all biholomorphic maps $h$ such that $h(M) \subset M$. If $(M, p)$ is finitely nondegenerate, then there exists $k$ such that the germ of the $k$-th Segre map at $p$ is a local holomorphic embedding. Then one can use the Implicit function Theorem to have the local holomorphic inverse of the $k$-th Segre map, which fails to hold if the $k$-th Segre map is merely finite to one. To construct a Basic identity of reflection type we use a meromorphic function defined in $\mathcal{M} \times{ }_{U} J^{k}(U, U)$, where $\mathcal{M}$ is a complexification of $M$ (see the definition in Section 1) and resolve the singularity of this meromorphic function. To author's knowledge, this is the first result of Basic identity of reflection type for CR manifolds which are not finitely nondegenerate. By the same arguments as in [16] using this basic identity, we prove the theorem.

Author thanks Dmitri Zaitsev for pointing out the optimality of the condition in Theorem 1 and giving the second example in Section 3.

\section{$\S 1$. Segre map}

Let $(M, 0)$ be the germ of a real analytic $\left(C^{\omega}\right)$ submanifold in $\mathbb{C}^{N}$ of codimension $d .(M, 0)$ is called generic if there exist a neighborhood $U$ of 0 
in $\mathbb{C}^{N}$ and a $C^{\omega}$ function $\rho=\left(\rho^{1}, \ldots, \rho^{d}\right)$ such that

$$
M=\{Z \in U: \rho(Z, \bar{Z})=0\}
$$

and $\partial \rho^{1} \wedge \cdots \wedge \partial \rho^{d} \neq 0$ on $M$. The CR structure of $M$ is defined by

$$
T^{1,0}(M):=T^{1,0}\left(\mathbb{C}^{N}\right) \cap \mathbb{C} T(M),
$$

where $T^{1,0}\left(\mathbb{C}^{N}\right)$ is the holomorphic tangent bundle over $\mathbb{C}^{N}$. It is known that any $C^{\omega} \mathrm{CR}$ manifold can be embedded into a complex space as a $C^{\omega}$ generic submanifold.

The complexification $\mathcal{M}$ of $M$ is defined by

$$
\mathcal{M}:=\{(Z, \xi) \in U \times \bar{U}: \rho(Z, \xi)=0\}
$$

after shrinking $U$ if necessary. For any point $p \in U$, the Segre variety $Q_{p}$ of $M$ at $p$ is a complex submanifold defined by

$$
Q_{p}:=\{Z \in U:(Z, \bar{p}) \in \mathcal{M}\} .
$$

Then $p \in Q_{p}$ if and only if $p \in M$ and $p \in Q_{q}$ if and only if $q \in Q_{p}$.

It is known that $Q_{p}$ is independent of the choice of the defining function $\rho$. Moreover, for any germ of a $C^{\omega}$ submanifold $\left(M^{\prime}, p^{\prime}\right)$ in $\mathbb{C}^{N^{\prime}}$ and for any germ of a holomorphic map $h:\left(\mathbb{C}^{N}, p\right) \rightarrow\left(\mathbb{C}^{N^{\prime}}, p^{\prime}\right)$ such that $h(M) \subset M^{\prime}$, we have $h\left(Q_{q}\right) \subset Q_{h(q)}^{\prime}$ for all $q$ sufficiently close to $p$, where $Q_{q^{\prime}}^{\prime}$ is the Segre variety of $M^{\prime}$ at $q^{\prime}$.

For the germ of a complex submanifold $(\Sigma, p)$ in $U$, we denote its $k$-jet at $p$ by $j_{p}^{k} \Sigma$. Also we denote the $k$-jet bundle of the germs of $n$-dimensional complex submanifolds in $U$ by $J^{k, n}(U)$.

Let $n:=N-d$. For any positive integer $k$, define $\pi_{M}^{k}: \mathcal{M} \rightarrow J^{k, n}(U)$ by

$$
\pi_{M}^{k}(Z, \xi):=\left(Z, j_{Z}^{k} Q_{\bar{\xi}}\right)
$$

It is easy to see that $\pi_{M}^{k}$ is holomorphic.

Definition 4. We call $\pi_{M}^{k}(0, \cdot): \overline{Q_{0}} \rightarrow J^{k, n}(U)$ the $k$-th Segre map at 0 .

Recall that a holomorphic mapping $\chi: \mathcal{V} \rightarrow \mathcal{W}$ between two holomorphic varieties is called a finite branched holomorphic covering if $\chi$ is a finite proper mapping and there is a proper subvariety $\mathcal{W}_{0}$ of $\mathcal{W}$ such that $\mathcal{W} \backslash \mathcal{W}_{0}$ 
is dense in $\mathcal{W}$ and $\chi: \mathcal{V} \backslash \pi^{-1}\left(\mathcal{W}_{0}\right) \rightarrow \mathcal{W} \backslash \mathcal{W}_{0}$ is a holomorphic covering map. The critical set $\mathcal{C}$ of a finite branched holomorphic covering $\chi$ is the set

$$
\mathcal{C}:=\left\{p \in \mathcal{V}: \operatorname{rank} \chi(p)<\operatorname{dim}_{p} \mathcal{V}\right\}
$$

Then $\mathcal{C}$ is a proper subvariety of $\mathcal{V}$.

Now suppose $M$ is essentially finite at 0 . Choose an integer $k$ such that the $k$-th Segre map at 0 is a finite branched holomorphic covering onto its image. Then by upper semi-continuity of the fiber dimension, we obtain the following lemma.

Lemma 5. Suppose that the critical set of the $k$-th Segre map at 0 is of codimension greater than or equal to 2 . Then for any $Z$ sufficiently close to 0 , the map $\pi_{M}^{k}(Z, \cdot): \overline{Q_{Z}} \rightarrow J^{k, n}(U)$ is a finite branched holomorphic covering onto its image with the critical set of codimension greater than or equal to 2.

\section{§2. Analytic jet parametrization for CR submersions}

Let $(M, 0)$ and $\left(M^{\prime}, 0\right)$ be the germs of $C^{\omega}$ generic submanifolds in $\mathbb{C}^{N}$ and $\mathbb{C}^{N^{\prime}}$ of codimension $d$ and $d^{\prime}$, respectively. The germ of a holomorphic map $f:\left(\mathbb{C}^{N}, 0\right) \rightarrow\left(\mathbb{C}^{N^{\prime}}, 0\right)$ is called $\mathrm{CR}$ submersion if $f(M) \subset M^{\prime}$ and $f_{*}\left(T_{0}^{1,0}(M)\right)=T_{0}^{1,0}\left(M^{\prime}\right)$.

Theorem 6. Let $(M, 0)$ and $\left(M^{\prime}, 0\right)$ be as above. Suppose $(M, 0)$ is of finite type and suppose there exists a positive integer $k_{0}$ such that the $k_{0}$-th Segre map of $M^{\prime}$ at 0 is a finite branched holomorphic covering onto its image with critical set of codimension greater than or equal to 2 at 0.

Then for any $C R$ submersion $f:\left(\mathbb{C}^{N}, 0\right) \rightarrow\left(\mathbb{C}^{N^{\prime}}, 0\right)$, there exist an integer $k$, a neighborhood $U$ of 0 in $\mathbb{C}^{N}$, a neighborhood $V$ of $\left(0, j_{0}^{k} f\right)$ in $J^{k}\left(\mathbb{C}^{N}, \mathbb{C}^{N^{\prime}}\right)$ and a holomorphic map $\Phi$ on $U \times V$ such that for any $p \in M \cap U$ and for any germ of a holomorphic map $h$ at $p$ such that $\left(p, j_{p}^{k} h\right) \in V$ and $h(M) \subset M^{\prime}$, we have

$$
h(Z)=\Phi\left(Z, p, j_{p}^{k} h\right)
$$

for all $Z$ sufficiently close to $p$. $k$ can be chosen independently of $f$ if $f$ is invertible.

Let $n:=N-d$ and $n^{\prime}:=N^{\prime}-d^{\prime}$. Choose local coordinates $(z, w) \in$ $\mathbb{C}^{n} \times \mathbb{C}^{d}$ and $\left(z^{\prime}, w^{\prime}\right) \in \mathbb{C}^{n^{\prime}} \times \mathbb{C}^{d^{\prime}}$ of $\mathbb{C}^{N}$ and $\mathbb{C}^{N^{\prime}}$ at the origin, respectively 
such that $T_{0}^{1,0}(M)=\left\{(z, w) \in \mathbb{C}^{N}: w=0\right\}$ and $T_{0}^{1,0}\left(M^{\prime}\right)=\left\{\left(z^{\prime}, w^{\prime}\right) \in\right.$ $\left.\mathbb{C}^{N^{\prime}}: w^{\prime}=0\right\}$. Assume that $f$ satisfies

$$
\operatorname{rank} \frac{\partial\left(f^{1}, \ldots, f^{n^{\prime}}\right)}{\partial\left(z_{1}, \ldots, z_{n^{\prime}}\right)}(0)=n^{\prime}
$$

where $f^{i}$ is the $i$-th component of $f$ with respect to $\left(z^{\prime}, w^{\prime}\right)$.

For the germ of an $n$-dimensional complex submanifold $(\Sigma, p)$ in $\mathbb{C}^{N}$ such that $T_{p} \Sigma$ is transversal to $\left\{(z, w) \in \mathbb{C}^{N}: z=0\right\}$, define

$$
\Sigma^{p}:=\left\{Z \in \Sigma: z_{n^{\prime}+1}=p_{n^{\prime}+1}, \ldots, z_{n}=p_{n}\right\} .
$$

Since $f$ satisfies $(2.1)$, the image $f\left(\Sigma^{p}\right) \subset \mathbb{C}^{N^{\prime}}$ is an $n^{\prime}$-dimensional complex submanifold if $p$ is sufficiently close to 0 . Moreover, if $\Sigma=Q_{0}$ and $f(M) \subset$ $M^{\prime}$, then $f\left(\Sigma^{0}\right)=Q_{0}^{\prime}$ in the sense of germ at 0 .

Let $U$ and $U^{\prime}$ be neighborhoods of 0 in $\mathbb{C}^{N}$ and $\mathbb{C}^{N^{\prime}}$, respectively such that there exist complexification $\mathcal{M}$ of $M$ and $\mathcal{M}^{\prime}$ of $M^{\prime}$ of the form (1.1). Now for $k \geq 1$, define $\phi^{k}$ by

$$
\phi^{k}\left(Z, \xi, j_{Z}^{k} h\right):=\left(h(Z), j_{h(Z)}^{k} h\left(Q \frac{Z}{\xi}\right)\right),
$$

where $(Z, \xi) \in \mathcal{M}$ and $\left(Z, j_{Z}^{k} h\right) \in J^{k}\left(U, U^{\prime}\right)$ such that

$$
\operatorname{rank} \frac{\partial\left(h^{1}, \ldots, h^{n^{\prime}}\right)}{\partial\left(z_{1}, \ldots, z_{n^{\prime}}\right)}(Z)=n^{\prime} .
$$

Then $\phi^{k}$ is a holomorphic map defined in a neighborhood of $\left(0,0, j_{0}^{k} f\right) \in$ $\mathcal{M} \times_{U} J^{k}\left(U, U^{\prime}\right)$. Moreover, if $h(M) \subset M^{\prime}$, then

$$
\pi_{M^{\prime}}^{k}(H(Z, \xi))=\phi^{k}\left(Z, \xi, j_{Z}^{k} h\right),
$$

where $H(Z, \xi)=(h(Z), \bar{h}(\xi))$.

Lemma 7. Let $k_{0}$ be as in Theorem 6. Let $\pi:=\pi_{M^{\prime}}^{k_{0}}$ and $\phi:=\phi^{k_{0}}$. Then there exist a neighborhood $W$ of $\left(0,0, j_{0}^{k_{0}} f\right)$ in $\mathcal{M} \times_{U} J^{k_{0}}\left(U, U^{\prime}\right)$, a variety $\mathcal{V}$ in $W$ and bounded maps $\phi_{s}: \mathcal{V} \rightarrow \mathcal{M}^{\prime}, s=1, \ldots, m$, for some integer $m$ such that the following conditions hold:

i) For any $p \in M$ sufficiently close to 0 and for any germ of a holomorphic map $h$ at $p$ such that $\left(p, \bar{p}, j_{p}^{k_{0}} h\right) \in W$ and $h(M) \subset M^{\prime}$, we have $\left(Z, \xi, j_{Z}^{k_{0}} h\right) \in \mathcal{V}$ for all $(Z, \xi) \in \mathcal{M}$ sufficiently close to $(p, \bar{p})$. 
ii) $\pi \circ \phi_{s}=\phi$ for all $s=1, \ldots, m$.

iii) $\phi_{s}, s=1, \ldots, m$, are holomorphic outside the singular locus of $\mathcal{V}$.

Proof. After shrinking $U$ if necessary, we can choose local coordinates $(Z, \zeta) \in \mathbb{C}^{N} \times \mathbb{C}^{n}$ of $\mathcal{M}$ at $(0,0)$ such that $\left\{(Z, \zeta) \in \mathbb{C}^{N} \times \mathbb{C}^{n}: Z \in U,|\zeta|<\right.$ $\epsilon\} \subset \mathcal{M}$ for a sufficiently small $\epsilon>0$ and for any $p \in U,\{(p, \zeta):|\zeta|<\epsilon\} \subset$ $\overline{Q_{p}}$. We use $\left(Z^{\prime}, \zeta^{\prime}\right) \in \mathbb{C}^{N^{\prime}} \times \mathbb{C}^{n^{\prime}}$ for $\mathcal{M}^{\prime}$ as local coordinates which have the same property as $(Z, \zeta)$ after shrinking $U^{\prime}$ if necessary. Also we use $(Z, \lambda)$ for $J^{k_{0}}\left(U, U^{\prime}\right)$ as local coordinates with $Z$ corresponding to base points and $\lambda$ corresponding to jets.

Since the $k_{0}$-th Segre map of $M^{\prime}$ at 0 is a finite branched holomorphic covering onto its image, by the definition of $\pi$, we can show that $\pi$ is a finite branched holomorphic covering at $(0,0)$ onto its image. Therefore $\pi\left(\mathcal{M}^{\prime}\right)$ is a variety at $\left(0, j_{0}^{k_{0}} Q_{0}^{\prime}\right)$.

Choose a neighborhood $W$ of $\left(0,0, j_{0}^{k_{0}} f\right) \in \mathcal{M} \times{ }_{U} J^{k_{0}}\left(U, U^{\prime}\right)$ in which $\phi$ is well-defined. Define

$$
\mathcal{A}:=\bigcap_{\zeta}\left\{(Z, \lambda) \in J^{k_{0}}\left(U, U^{\prime}\right):(Z, \zeta, \lambda) \in W, \phi(Z, \zeta, \lambda) \in \pi\left(\mathcal{M}^{\prime}\right)\right\}
$$

Since $\pi\left(\mathcal{M}^{\prime}\right)$ is a variety, $\mathcal{A}$ is a variety in a neighborhood of $\left(0, j_{0}^{k_{0}} f\right) \in$ $J^{k_{0}}\left(U, U^{\prime}\right)$. If $\left(Z, j_{Z}^{k_{0}} h\right) \in \mathcal{A}$, then $\phi\left(Z, \zeta, j_{Z}^{k_{0}} h\right) \in \pi\left(\{h(Z)\} \times \overline{Q_{h(Z)}^{\prime}}\right)$ for all sufficiently small $\zeta$. Moreover, by (2.2) we can show that if $h$ is a CR submersion at $p \in U$ such that $\left(p, j_{p}^{k_{0}} h\right) \in W$, then $\left(Z, j_{Z}^{k_{0}} h\right) \in \mathcal{A}$ for all $Z$ sufficiently close to $p$.

For $(Z, \lambda) \in \mathcal{A}$, define $\phi_{(Z, \lambda)}(\zeta):=\phi(Z, \zeta, \lambda)$. Since $f$ satisfies $(2.1)$, if $\lambda=j_{0}^{k_{0}} f$, then $\phi_{(0, \lambda)}$ is a surjective map onto $\pi\left(\{0\} \times \overline{Q_{0}^{\prime}}\right)$ and therefore by the assumption on the critical set $\mathcal{C}_{0}^{\prime}$ of the $k_{0}$-th Segre map of $M^{\prime}$ at 0 , the pre-image of $\pi\left(\mathcal{C}_{0}^{\prime}\right)$ under $\phi_{(0, \lambda)}$ is of codimension greater than or equal to 2. Moreover, by upper semi-continuity of the dimension, for all $(Z, \lambda) \in \mathcal{A}$ sufficiently close to $\left(0, j_{0}^{k_{0}} f\right)$, we have

$$
\operatorname{dim} \phi_{(Z, \lambda)}^{-1}\left(\mathcal{B}^{\prime}\right) \leq n-2,
$$

where $\mathcal{B}^{\prime}$ is the image of the critical set of $\pi$.

Choose a point $\zeta_{0}$ such that $\phi\left(0, \zeta_{0}, j_{0}^{k_{0}} f\right) \notin \mathcal{B}^{\prime}$. Since $\mathcal{M}^{\prime}$ is a connected complex manifold, $\pi: \mathcal{M}^{\prime} \backslash \pi^{-1}\left(\mathcal{B}^{\prime}\right) \rightarrow \pi\left(\mathcal{M}^{\prime}\right) \backslash \mathcal{B}^{\prime}$ is an $m$-sheeted holomorphic covering for some integer $m$. Hence there exist $m$ mutually distinct points $\zeta_{0,1}^{\prime}, \ldots, \zeta_{0, m}^{\prime}$ such that $\pi\left(0, \zeta_{0, s}^{\prime}\right)=\phi\left(0, \zeta_{0}, j_{0}^{k_{0}} f\right), s=1, \ldots, m$. 
Choose mutually disjoint neighborhoods $V_{s}^{\prime}, s=1, \ldots, m$, of $\left(0, \zeta_{0, s}^{\prime}\right)$ in $\mathcal{M}^{\prime} \backslash \pi^{-1}\left(\mathcal{B}^{\prime}\right)$ such that $\pi: V_{s}^{\prime} \rightarrow \pi\left(V_{1}^{\prime}\right)$ is biholomorphic for all $s=1, \ldots, m$.

After shrinking $W$ if necessary, we may assume that $\phi\left(Z, \zeta_{0}, \lambda\right) \in \pi\left(V_{1}^{\prime}\right)$ for all $(Z, \lambda) \in \mathcal{A}$. Since $\operatorname{dim} \phi_{(Z, \lambda)}^{-1}\left(\mathcal{B}^{\prime}\right) \leq n-2$ for all $(Z, \lambda)$ sufficiently close to $\left(0, j_{0}^{k_{0}} f\right), \overline{Q_{Z}} \backslash \phi_{(Z, \lambda)}^{-1}\left(\mathcal{B}^{\prime}\right)$ is simply connected. Then after shrinking $U$ and $W$ if necessary, for all $(Z, \lambda) \in \mathcal{A}$, we can choose $m$ distinct holomorphic lifts

$$
\phi_{s,(Z, \lambda)}: \overline{Q_{Z}} \backslash \phi_{(Z, \lambda)}^{-1}\left(\mathcal{B}^{\prime}\right) \longrightarrow \mathcal{M}^{\prime} \backslash \pi^{-1}\left(\mathcal{B}^{\prime}\right), \quad s=1, \ldots, m,
$$

of $\phi_{(Z, \lambda)}$ such that $\phi_{s,(Z, \lambda)}\left(\zeta_{0}\right) \in V_{s}^{\prime}$. Since $\phi_{s,(Z, \lambda)}$ is bounded and holomorphic in $\overline{Q_{Z}} \backslash \phi_{(Z, \lambda)}^{-1}\left(\mathcal{B}^{\prime}\right), \phi_{s,(Z, \lambda)}$ extends holomorphically to $\overline{Q_{Z}}$.

Now define

$$
\mathcal{V}:=\{(Z, \zeta, \lambda) \in W:(Z, \lambda) \in \mathcal{A}\}
$$

and define

$$
\phi_{s}: \mathcal{V} \longrightarrow \mathcal{M}^{\prime}
$$

by $\phi_{s}(Z, \zeta, \lambda):=\phi_{s,(Z, \lambda)}(\zeta)$.

Claim 8. For each $s, \phi_{s}: \mathcal{V}_{r} \rightarrow \mathcal{M}^{\prime}$ is holomorphic, where $\mathcal{V}_{r}$ is the set of regular points of $\mathcal{V}$.

Proof. Let

$$
\mathcal{S}:=\left\{(Z, \zeta, \lambda) \in \mathcal{V}:(Z, \lambda) \in \mathcal{A}_{\sigma}\right\} \cup\left(\phi^{-1}\left(\mathcal{B}^{\prime}\right) \cap \mathcal{V}\right),
$$

where $\mathcal{A}_{\sigma}$ is the singular locus of $\mathcal{A}$. Then $\mathcal{S}$ is a proper subvariety of $\mathcal{V}$ such that $\mathcal{V} \backslash \mathcal{S}$ is dense in $\mathcal{V}_{r}$

Let $(Z, \zeta, \lambda) \in \mathcal{V} \backslash \mathcal{S}$. Choose a curve $c:[0,1] \rightarrow \mathbb{C}^{n}$ such that $c(0)=\zeta_{0}$, $c(1)=\zeta$ and $\gamma(t):=(Z, c(t), \lambda) \in \mathcal{V} \backslash \mathcal{S}$ for all $t \in[0,1]$. Let $\gamma^{\prime}(t):=\phi(\gamma(t))$ and $\widetilde{\gamma}(t):=\phi_{s}(\gamma(t))$. Since $\widetilde{\gamma}([0,1])$ is compact and $\pi: \mathcal{M}^{\prime} \backslash \pi^{-1}\left(\mathcal{B}^{\prime}\right) \rightarrow$ $\pi\left(\mathcal{M}^{\prime}\right) \backslash \mathcal{B}^{\prime}$ is a topological covering map, there exists a tubular neighborhood $W_{\widetilde{\gamma}}$ of $\widetilde{\gamma}([0,1])$ such that $\pi\left(W_{\widetilde{\gamma}}\right)$ is an open neighborhood of $\gamma^{\prime}$ in $\pi\left(\mathcal{M}^{\prime}\right) \backslash \mathcal{B}^{\prime}$ and any curve in $\pi\left(W_{\widetilde{\gamma}}\right)$ which is homotopic to $\gamma^{\prime}$ has a unique lift in $W_{\widetilde{\gamma}}$.

Since $\phi$ is holomorphic in $\mathcal{V} \backslash \mathcal{S}$ and $\gamma([0,1])$ is compact, there exists a contractible neighborhood $W_{\mathcal{A}}$ of $(Z, \lambda)$ in $\mathcal{A} \backslash \mathcal{A}_{\sigma}$ such that for all $(\tilde{Z}, \tilde{\lambda}) \in$ $W_{\mathcal{A}}$,

$$
\{(\tilde{Z}, c(t), \tilde{\lambda}): t \in[0,1]\} \subset \mathcal{V} \backslash \mathcal{S}
$$

and

$$
\{\phi(\tilde{Z}, c(t), \tilde{\lambda}): t \in[0,1]\} \subset \pi\left(W_{\widetilde{\gamma}}\right) .
$$


Then the curve $t \rightarrow \phi(\tilde{Z}, c(t), \tilde{\lambda})$ is homotopic to $\gamma^{\prime}$ and hence has a unique lift $t \rightarrow \phi_{s}(\tilde{Z}, c(t), \tilde{\lambda})$ in $W_{\tilde{\gamma}}$. Thus there exists a neighborhood of $(Z, \zeta, \lambda)$ such that $\phi_{s}=\pi^{-1} \circ \phi$ in it, where $\pi^{-1}$ is a local inverse of $\pi$ in a neighborhood of $\phi(Z, \zeta, \lambda)$.

Since $\pi$ is a finite branched holomorphic covering and $\phi$ is holomorphic, $\phi_{s}$ is holomorphic in $\mathcal{V} \backslash \mathcal{S}$. Since $\mathcal{V}_{r}$ is a complex manifold and $\phi_{s}$ is bounded, $\phi_{s}$ extends holomorphically to $\mathcal{V}_{r}$.

Then $W, \mathcal{V}$ and $\phi_{s}, s=1, \ldots, m$, satisfy the required properties.

Lemma 9. Let $W, \mathcal{V}$ and $\phi_{s}, s=1, \ldots, m$, be as in Lemma 7. Then there exist holomorphic functions $\psi_{s}, s=1, \ldots, m$, defined in a neighborhood $W_{1} \subset W$ of $\left(0,0, j_{0}^{k_{0}} f\right)$ such that $\psi_{s} \equiv \phi_{s}$ on $\mathcal{V} \cap W_{1}$ for all $s=1, \ldots, m$.

Proof. Since $\phi_{s}, s=1, \ldots, m$, are bounded and holomorphic outside the singular locus of $\mathcal{V}$, by Theorem P.9 of [11], each component of $\phi_{s}$ extends meromorphically to a neighborhood of $\mathcal{V}$. Let $\phi_{s}^{i}$ be the $i$-th component of $\phi_{s}$ with respect to the coordinates $\left(Z^{\prime}, \zeta^{\prime}\right)$ and let $\mathcal{S}\left(\phi_{s}^{i}\right)$ be the singular variety of $\phi_{s}^{i}$, i.e. the set of points at which $\phi_{s}^{i}$ is not holomorphic.

Since $\phi_{s}^{i}$ is holomorphic outside $\phi^{-1}\left(\mathcal{B}^{\prime}\right)$ and $\phi_{\left(0, j_{0}^{k_{0}} f\right)}^{-1}\left(\mathcal{B}^{\prime}\right)$ is a proper subvariety of $\{0\} \times \overline{Q_{0}}$, we can choose a holomorphic function $\eta$ such that $\eta\left(0, \zeta, j_{0}^{k_{0}} f\right) \not \equiv 0$ and vanishes on $\mathcal{S}\left(\phi_{s}^{i}\right)$. By Theorem P.7 of [11], there is an integer $l \geq 1$ such that $\eta^{l} \phi_{s}^{i}$ is holomorphic in a neighborhood of $\left(0,0, j_{0}^{k_{0}} f\right) \in \mathcal{M} \times_{U} J^{k_{0}}\left(U, U^{\prime}\right)$. We replace $\eta$ with $\eta^{l}$ and denote it by $\eta$ again.

Let $\phi_{s}^{i}=\mu / \eta$, where $\mu$ is a holomorphic function in a neighborhood of $\left(0,0, j_{0}^{k_{0}} f\right)$. Since $\eta\left(0, \cdot, j_{0}^{k_{0}} f\right) \not \equiv 0$, by Weierstrass Preparation Theorem we may assume that $\eta$ is a Weierstrass polynomial in $\zeta_{1}$. By Weierstrass Division Theorem, we have

$$
\mu=\psi_{s}^{i} \eta+r,
$$

where $\psi_{s}^{i}$ is a holomorphic function in a neighborhood of $\left(0,0, j_{0}^{k_{0}} f\right)$ and $r$ is a polynomial in $\zeta_{1}$ with coefficients which are holomorphic in other variables such that the degree in $\zeta_{1}$ is strictly less than that of $\eta$.

Fix $(Z, \lambda) \in \mathcal{A}$. Then $\phi_{s}(Z, \zeta, \lambda) \in \mathcal{M}^{\prime}$ for all $\zeta \in \overline{Q_{Z}}$. Hence

$$
\phi_{s}^{i}(Z, \zeta \lambda)=\psi_{s}^{i}(Z, \zeta, \lambda)+\frac{r(Z, \zeta, \lambda)}{\eta(Z, \zeta, \lambda)}
$$


should be bounded for all $\zeta \in \mathbb{C}^{n}$ with $|\zeta|<\epsilon$.

On the other hand, $r(Z, \zeta, \lambda) / \eta(Z, \zeta, \lambda)$ is a rational function in $\zeta_{1}$ with $\operatorname{deg}(r)<\operatorname{deg}(\eta)$. Therefore $r(Z, \cdot, \lambda) / \eta(Z, \cdot, \lambda)$ is bounded if and only if $r(Z, \cdot, \lambda) \equiv 0$. Hence we obtain

$$
\phi_{s}^{i}(Z, \zeta, \lambda)=\psi_{s}^{i}(Z, \zeta, \lambda)
$$

if $(Z, \zeta, \lambda) \in \mathcal{V}$.

Now let $\psi_{s}$ be the holomorphic map with $\psi_{s}^{i}$ as its $i$-th component for all $i$ and extend $\psi_{s}$ to a neighborhood of $\left(0,0, j_{0}^{k_{0}} f\right) \in(U \times \bar{U}) \times_{U} J^{k_{0}}\left(U, U^{\prime}\right)$. By construction, we have $\psi_{s}(Z, \zeta, \lambda)=\phi_{s}(Z, \zeta, \lambda)$ if $(Z, \zeta, \lambda) \in \mathcal{V}$.

Proof of Theorem 6. Let $h$ be the germ of a holomorphic map at $p \in M$ such that $\left(p, \bar{p}, j_{p}^{k_{0}} h\right) \in W_{1}$ and $h(M) \subset M^{\prime}$. Then by i) and ii) of Lemma 7 and by Lemma 9 , there exists $s$ such that

$$
H(Z, \xi)=\psi_{s}\left(Z, \xi, j_{Z}^{k_{0}} h\right)
$$

for all $(Z, \xi) \in \mathcal{M}$ sufficiently close to $(p, \bar{p})$.

By the construction of $\psi_{s}$, we have $\psi_{s}\left(0, \cdot, j_{0}^{k_{0}} f\right) \not \equiv \psi_{s^{\prime}}\left(0, \cdot, j_{0}^{k_{0}} f\right)$ if $s \neq s^{\prime}$. Since the map $\xi \rightarrow \psi_{s}\left(0, \xi, j_{0}^{k_{0}} f\right)$ is holomorphic in $\xi$, there is an integer $k_{1}$ such that the $k_{1}$-jets of the maps $\xi \rightarrow \psi_{s}\left(0, \xi, j_{0}^{k_{0}} f\right), s=1, \ldots, m$, at 0 are mutually distinct. Assume that $k_{1} \geq k_{0}$. We regard $\psi_{s}$ as a function defined in a neighborhood of $\left(0,0, j_{0}^{k_{1}} f\right) \in(U \times \bar{U}) \times_{U} J^{k_{1}}\left(U, U^{\prime}\right)$.

Assume that the $k_{1}$-th jet of the map $\xi \in \overline{Q_{0}} \rightarrow \bar{f}(\xi)$ at 0 is given by the $k_{1}$-th jet of the map $\xi \rightarrow \psi_{1}\left(0, \xi, j_{0}^{k_{0}} f\right)$. Choose neighborhoods $U_{1} \subset U$ of 0 and $V \subset J^{k_{1}}\left(U, U^{\prime}\right)$ of $\left(0, j_{0}^{k_{1}} f\right)$ such that $\psi_{s}, s=1, \ldots, m$, are holomorphic in $\left(U_{1} \times \bar{U}_{1}\right) \times_{U_{1}} \times V$ and if $h$ is a germs of a holomorphic map at $p \in M \cap U_{1}$ such that $\left(p, j_{p}^{k_{1}} h\right) \in V$ and $h(M) \subset M^{\prime}$, then

$$
H(p, \xi)=\psi_{1}\left(p, \xi, j_{p}^{k_{0}} h\right)
$$

for all $\xi \in \overline{Q_{p}}$ sufficiently close to $\bar{p}$. Since $\psi_{1}$ is a lifting of $\phi$ on $\mathcal{V}$, by the uniqueness of lifting, we obtain

$$
H(Z, \xi)=\psi_{1}\left(Z, \xi, j_{Z}^{k_{0}} h\right)
$$

for all $(Z, \xi) \in \mathcal{M}$ sufficiently close to $(p, \bar{p})$.

We regard $\psi_{1}$ as a function defined in $\mathcal{M} \times_{U} J^{k_{1}}\left(\mathbb{C}^{N}, \mathbb{C}^{N^{\prime}}\right)$. Then by Proposition 5.4 in Section 4 of [16] with (2.4) as basic identity of reflection 
type, we can construct a holomorphic map $\Phi$ defined in a neighborhood of $\left(0,0, j^{(2 d+1) k_{1}} f\right)$ such that for any germ of a holomorphic map $h$ at $p$ such that $\left(p, j_{p}^{(2 d+1) k_{1}} h\right)$ is sufficiently close to $\left(0, j_{0}^{(2 d+1) k_{1}} f\right)$ and $h(M) \subset M^{\prime}$, we have

$$
h(Z)=\Phi\left(Z, p, j_{p}^{(2 d+1) k_{1}} h\right)
$$

for all $Z$ sufficiently close to $p$.

Proof of Corollary 2. Let $p=0$ and $(M, 0)=\left(M^{\prime}, 0\right)$. Let $k$ be the integer given in Theorem 6 corresponding to the identity map. Then it is easy to see that

$$
j_{0}^{k}: \operatorname{Aut}(M, 0) \longrightarrow G_{0}^{k}\left(\mathbb{C}^{N}\right)
$$

is injective and continuous.

Let $\left\{\lambda_{j}\right\} \subset j_{0}^{k}(\operatorname{Aut}(M, 0))$ be a sequence converging to $\lambda \in j_{0}^{k}(\operatorname{Aut}(M, 0))$. Choose $h_{j} \in \operatorname{Aut}(M, 0)$ and $h \in \operatorname{Aut}(M, 0)$ such that $j_{0}^{k} h_{j}=\lambda_{j}$ for all $j$ and $j_{0}^{k} h=\lambda$. We may assume that $h=$ id. Let $\Phi$ be the holomorphic map given in Theorem 6 corresponding to the identity map. Then

$$
h_{j}(Z)=\Phi\left(Z, 0, \lambda_{j}\right)
$$

for all sufficiently large $j$ and

$$
h(Z)=\Phi(Z, 0, \lambda) .
$$

Since $\lambda_{j} \rightarrow \lambda, h_{j}$ converges uniformly to $h$ on a compact neighborhood of 0 . Hence $j_{0}^{k}: \operatorname{Aut}(M, 0) \rightarrow j_{0}^{k}(\operatorname{Aut}(M, 0))$ is a homeomorphism.

Now we show that $j_{0}^{k}(\operatorname{Aut}(M, 0))$ is totally real in $G_{0}^{k}\left(\mathbb{C}^{N}\right)$. Let $\Phi$ be as above. Then for any $h \in \operatorname{Aut}(M, 0)$ such that $\left(0, j_{0}^{k} h\right)$ is sufficiently closed to $\left(0, j_{0}^{k} \mathrm{id}\right)$, we have

$$
h(Z)=\Phi\left(Z, 0, j_{0}^{k} h\right)
$$

Then by differentiating (2.5) with respect to $Z$ and evaluating $Z=0$, for any integer $l$, we have

$$
j_{0}^{l} h=\Psi^{l}\left(j_{0}^{k} h\right)
$$

for some holomorphic map $\Psi^{l}$.

Let $\psi$ be a holomorphic map as in Lemma 9 such that

$$
\operatorname{id}(Z, \xi)=\psi\left(Z, \xi, j_{Z}^{k_{0}} \mathrm{id}\right)
$$


and let $k_{1} \leq k$ be an integer as in the proof of Theorem 6 such that for any $h \in \operatorname{Aut}(M, 0)$ such that $\left(Z, j_{Z}^{k_{1}} h\right)$ is sufficiently close to $\left(0, j_{0}^{k_{1}} \mathrm{id}\right), h$ satisfies

$$
H(Z, \xi)=\psi\left(Z, \xi, j_{Z}^{k_{0}} h\right)
$$

for all $(Z, \xi) \in \mathcal{M}$ sufficiently close to $(0,0)$.

By differentiating (2.6) with respect to $\xi$ and evaluating $Z=\xi=0$, we have

$$
j_{0}^{k} \bar{h}=\Gamma\left(j_{0}^{k_{0}+k} h\right)
$$

for some holomorphic map $\Gamma$. By substituting $\Psi^{k_{0}+k}\left(j_{0}^{k} h\right)$ for $j_{0}^{k_{0}+k} h$ we have

$$
j_{0}^{k} \bar{h}=\Gamma\left(\Psi^{k_{0}+k}\left(j_{0}^{k} h\right)\right) .
$$

Hence $j_{0}^{k}(\operatorname{Aut}(M, 0))$ is totally real in $G_{0}^{k}\left(\mathbb{C}^{N}\right)$.

\section{§3. Examples}

In this section we give two examples. First example is to show that finite nondegeneracy is stronger than the condition given in Theorem 1 . The second example is to show that the condition in Theorem 1 is essential to have an analytic jet parametrization.

Let

$$
M:=\left\{\left(z_{1}, z_{2}, w\right) \in \mathbb{C}^{3}:\left|z_{1}\right|^{2}+\left(\left|z_{1}\right|^{2}+\left|z_{2}\right|^{2}\right)\left|z_{2}\right|^{2}+|w|^{2}=1\right\} .
$$

We will show that $M$ satisfies the condition on the codimension of the critical set of the Segre map given in Theorem 1 but is not finitely nondegenerate at $(0,0, w)$ with $|w|=1$. Moreover, by computing the Levi form, we can show that $M$ is strictly pseudoconvex at any $p=\left(z_{1}, z_{2}, w\right) \in M$ with $|w| \neq 1$.

Since $M$ has a CR automorphism $\left(z_{1}, z_{2}, w\right) \rightarrow\left(a_{1} z_{1}, a_{2} z_{2}, a_{3} w\right)$ for some $a_{i} \in \mathbb{C}$ with $\left|a_{i}\right|=1$, it is enough to show the above for $(0,0,1)$. Let $p=(0,0,1)$. Then $\overline{Q_{p}}=\left\{\left(z_{1}, z_{2}, 1\right): z_{i} \in \mathbb{C}\right\}$ and the $k$-th Segre map is given by

$$
j_{p}^{k}\left(p,\left(z_{1}, z_{2}, 1\right)\right)=\left(p, z_{1}, z_{1} z_{2}, 2 z_{2}^{2}, 0, \ldots\right)
$$

after reordering coordinates, where ... are all constants. Thus the critical set is $\{(0,0,1)\}$ whose codimension is 2 and $M$ is not finitely nondegenerate at $(0,0,1)$. 
For the second example, let

$$
M=\left\{(z, w) \in \mathbb{C}^{2}: \Im w=|z|^{4}\right\} .
$$

We can show that $M$ is essentially finite at 0 and for any $k$, the codimension of the critical set of the $k$-th Segre map at 0 is 1 . For any point $p=(z, w) \in$ $M, M$ is locally biholomorphically equivalent to the unit sphere $S^{3}$ in $\mathbb{C}^{2}$ unless $z=0$. Hence in this case $\operatorname{Aut}(M, p)$ is isomorphic to the stability group of $S^{3}$ unless $z=0$. On the other hand, any $h \in \operatorname{Aut}(M, 0)$ is of the form

$$
h(z, w)=\left(\frac{a z}{(1+r w)^{1 / 2}}, \frac{|a|^{4} w}{1+r w}\right),
$$

where $a \in \mathbb{C}-\{0\}, r \in \mathbb{R}$ and $(1+\zeta)^{1 / 2}$ is the square root of $(1+\zeta)$ with principal branch cut. Therefore

$$
\operatorname{dim} \operatorname{Aut}(M, 0)<\operatorname{dim} \operatorname{Aut}(M, p)
$$

for all $p=(z, w)$ such that $z \neq 0$. Suppose there exists an analytic jet parametrization of the form (0.1). Then the image $\left\{j_{p}^{k} f, p \in M, f \in\right.$ Aut $(M, p)\}$ is an analytic set. Therefore the dimension of the fiber $\left\{j_{p}^{k} f, f \in\right.$ $\operatorname{Aut}(M, p)\}$ is a lower semi-continuous function as $p$ varies, which is incompatible with (3.1).

\section{REFERENCES}

[1] M. S. Baouendi, P. Ebenfelt and L. P. Rothschild, Algebraicity of holomorphic mappings between real algebraic sets in $\mathbb{C}^{n}$, Acta Math., 177 (1996), 225-273.

[2] M. S. Baouendi, P. Ebenfelt and L. P. Rothschild, Parametrization of local biholomorphisms of real analytic hypersurfaces, Asian J. Math., 1 (1997), 1-16.

[3] M. S. Baouendi, P. Ebenfelt and L. P. Rothschild, CR automorphisms of real analytic manifolds in complex space, Comm. Anal. geom., 6 (1998), 291-315.

[4] M. S. Baouendi, P. Ebenfelt and L. P. Rothschild, Real submanifolds in complex space and their mappings, Princeton University Press, Princeton, New Jersey, 1999.

[5] M. S. Baouendi, P. Ebenfelt and L. P. Rothschild, Rational dependence of smooth and analytic CR mappings on their jets, Math. Ann., 315 (1999), 205-249.

[6] M. S. Baouendi, P. Ebenfelt and L. P. Rothschild, Convergence and finite determination of formal CR mappings, J. Amer. Math. Soc., 13 (2000), 697-723.

[7] M. S. Baouendi, L. P. Rothschild, J. Winkelmann and D. Zaitsev, Lie group structures on groups of diffeomorphisms and applications to CR manifolds, Ann. de L'Institut Fourier, 54 (2004), 1279-1303. 
[8] T. Bloom and I. Graham, On type conditions for generic real submanifolds of $\mathbb{C}^{n}$, Invent. Math., 40 (1977), 217-243.

[9] S. S. Chern and J. K. Moser, Real hypersurfaces in complex manifolds, Acta Math., 133 (1974), 219-271.

[10] P. Ebenfelt, Finite jet determination of holomorphic mappings at the boundary, Asian J. Math., 5 (2001), 637-662.

[11] R. C. Gunning, Introduction to holomorphic functions of several variables, Vol. 2, Wordsworth and Brooks/Cole, Belmont, California, 1990.

[12] C. K. Han, Analyticity of $C R$ equivalences between some real hypersurfaces in $\mathbb{C}^{n}$ with degenerate Levi forms, Invent. Math., 73 (1983), 51-69.

[13] C. K. Han, Complete differential system for the mappings of CR manifolds of nondegenerate Levi forms, Math. Ann., 309 (1997), 401-409.

[14] A. Hayashimoto, On the complete system of finite order for CR mappings and its application, Osaka J. of Math., 35 (1998), 617-628.

[15] S.-Y. Kim, Complete system of finite order for CR mappings between real analytic hypersurfaces of degenerate Levi form, J. Korean math. Soc., 38 (2001), 87-99.

[16] S.-Y. Kim and D. Zaitsev, The equivalence and embedding problems for CR structures of any codimension, Topology, 44 (2005), 557-584.

[17] J. J. Kohn, Boundary behavior of $\bar{\partial}$ on weakly pseudo-convex manifolds of dimension two, J. Differential Geom., 6 (1972), 523-542.

[18] B. Lamel, Holomorphic maps of real submanifolds in complex spaces of different dimensions, Pacific J. Math., 201 (2001), 357-387.

[19] B. Lamel and N. Mir, Parametrization of local CR automorphisms by finite jets and applications, J. Amer. Math. Soc., to appear.

[20] S. Webster, On the reflection principle in several complex variables, Proc. Amer. Math. Soc., 71 (1978), 26-28.

[21] D. Zaitsev, Germs of local automorphism of real analytic CR structures and analytic dependence on k-jets, Math. Research Letters, 4 (1997), 823-842.

Department of Mathematics Education

Kangwon National University

123 Hyoja-dong

Chuncheon

Kangwon-do, 200-701

Korea

sykim87@kangwon.ac.kr 